\title{
Translational Sleep Science in Behavioral Medicine: Introduction to this Special Issue
}

\author{
Natasha Williams ${ }^{1} \cdot$ Tracy Trevorrow $^{2}$ \\ Accepted: 30 December 2020 / Published online: 10 February 2021 \\ (c) International Society of Behavioral Medicine 2021
}

\begin{abstract}
The consideration of sleep and circadian rhythms in the context of health is a relatively recent development in the history of the field of behavioral medicine. This special issue of the International Journal of Behavioral Medicine recognizes that sleep and circadian rhythms are fundamental to appreciating physiological, psychological, social, and environmental factors in the health and well-being of the population. The articles included in this issue draw attention to the breadth and saliency of sleep as a marker of health status and as a target of behavioral intervention to promote health. Such research highlights the diversity of participants, research methods, and clinical significance of translational sleep science allowing us to recognize the role of sleep in the context of health in new ways. These studies also illustrate progress in integrating theory, employing prospective and longitudinal designs and multimodal and integrative assessments. This introduction to the special issue concludes by discussing challenges and opportunities in the field of behavioral sleep medicine, including those posed by the coronavirus disease 2019 (COVID-19) pandemic and the need to more effectively provide sleep disorder treatment among underserved populations.
\end{abstract}

Keywords Sleep $\cdot$ Circadian rhythms $\cdot$ Behavioral medicine $\cdot$ Coronavirus

\section{Introduction}

Over the past two decades, the number of published research studies that focus on sleep has increased seven-fold [1]; sleep has become a consistent topic of interest for behavioral medicine researchers and practitioners. The International Journal of Behavioral Medicine has included articles focused on sleep for each of the past 15 years. In 2016 the Society of Behavioral Medicine established a Special Interest Group dedicated to Sleep, which is serving as a co-sponsor of this special issue. The importance of sleep is well-established internationally in the behavioral medicine landscape, and this special issue serves to recognize this development, as well as to advance translational sleep science.

Natasha Williams

Natasha.williams2@nyulangone.org

1 NYU Grossman School of Medicine Division of Health and Behavior Center for Healthful Behavior Change, NYU, Langone Health, New York, NY, USA

2 Center for Medical Psychology, Chaminade University Honolulu, Honolulu, HI 96816, USA
The arguments for appreciating the role of sleep in behavioral medicine are clear. Sleep is influenced by behavioral, psychological, educational, and environmental factors, as well as community and organizational policies [2]. At the same time, sleep has mediating and moderating influences on health. Sleep has been extensively studied regarding its relationship to disorders that have been the focus of behavioral medicine, such as physical activity [3], pain [4], obesity [5], substance use [6], and chronic illness [7]. As such, behavioral sleep science spans a broad range of disciplines, as the nature of sleep and circadian rhythms requires an appreciation from biological, psychological, social, and environmental perspectives.

Accompanying our increased understanding of the importance of sleep is the realization that so many of us do not sleep well. Across the spectrum of sleep disorders, the most common sleep problem is insomnia [8]. A 2019 poll of US adults reported $84 \%$ complained of inadequate sleep at least once per week, and about half of adults surveyed reported excessive daytime fatigue 3 to 7 days per week [9]. Approximately 10 to $20 \%$ of patients in primary care centers complain of insomnia symptoms [8]. Obstructive apnea is also frequently observed in older populations and becoming 
increasingly prevalent in the general population due to rising rates of obesity. Prevalence rates for obstructive apnea are estimated at $17 \%$ for men and $9 \%$ for women over the age of 50 [10]. Inadequate sleep is not the sole providence of adults. School-aged children, particularly in the USA, have been systematically sleep deprived by inappropriately early school start times [11]. The US Center's for Disease Control and Prevention estimated that $60 \%$ of US middle school students and $70 \%$ of high school students do not get adequate sleep [12]. Currently, the coronavirus disease 2019 (COVID19) pandemic has worsened sleep across the globe. Multiple pandemic-related factors including psychological distress, social isolation, poor sleep hygiene, and decreased physical activity appear to have resulted in disordered sleep [13-15]. Pandemic-related sleep disruption has been reported to be greater in health care workers, youth, women, and those with low levels of social capital [14-17]. As such, there is great potential in promoting community health through sleep health education, policy changes (such as establishing later school start times) and identifying and providing therapies to communities at high risk for inadequate sleep.

\section{About This Issue}

It is a privilege to publish this special issue in the International Journal of Behavioral Medicine. We received an overwhelming response to the initial call for manuscripts. The editorial challenge of reducing over 50 submissions to a single issue was daunting! We could not include many worthy submissions in this issue, so we are delighted to report that a second special sister-issue, dedicated to sleep, is planned and forthcoming.

The purpose of this special issue is to showcase the breadth of sleep-related topics, the range of methodologies for appreciating the role of sleep in behavioral health, and the potential for significant health gains from sleep interventions. The selected papers for this special issue have clear theoretical frameworks, are grounded in existing literature, and employ strong designs. We were mindful to select studies that allow us to see the interrelationships between sleep and health in new ways. Consistent with the field of behavioral medicine, there is an applied focus of the selected articles. These papers increase the scientific and public understanding of the role of sleep in health and have implications for clinical and policy interventions to improve the health and wellbeing of individuals, families, communities, and populations. Participants in these studies span the developmental spectrum from children to older adults, include minority and underserved populations, and include studies conducted outside the continental USA. Sleep is not a unitary construct and researchers employ a broad range of approaches to assess sleep duration, efficiency, quality, and timing. Technologies are also employed to assess sleep behavior (e.g., movement monitors, such as actigraphs) and physiological processes associated with sleep. The assessment approaches in this special issue include self-report measures (surveys, self-monitoring diaries), actigraphs, and structured interviews.

\section{The Articles}

Papers in this issue fall into four general areas. Most studies focused on the effects of stress on sleep. Several papers examined the consequences of a stressful life role on sleep. Such roles included being a family member or other informal caregiver for those with cancer. Panjwani and colleagues ([18]; this issue) surveyed parents of teens and young adult children with cancer, focusing on parents' abilities to tolerate uncertainty. Being a graduate student is putatively understood to be stressful, yet, there are few published accounts of the sleep (or lack of sleep) of this population. Allen and colleagues ([19]; this issue) assessed 2683 graduate students to help fill this void and explored the phenomena of graduate school burnout. Bunjo and colleagues ([20]; this issue) considered the extent to which sleep duration moderated the relationship between work-life interference and time strain, work-to-community interactions, satisfaction with overall work-life balance, and depressive symptoms. Other papers in this group investigated the consequence to sleep after discrete stressful life events, such as job loss. Haynes and colleagues ([21]; this issue) evaluated recently unemployed individuals, finding connections between the type of work stress prior to job loss and long-term sleep problems once unemployed. Another study looked at the stress of pregnancy and the role of sleep in predicting newborn health status. Okun and colleagues ([22]; this issue) sought to determine whether sleep disruption early in pregnancy was implicated in inflammatory processes (cytokine levels) or newborn health outcomes.

A second group of studies investigated the moderating and mediating roles of sleep on disease and psychological maladies. Griffin and colleagues ([23]; this issue) examined the role of sleep as a mediator of the relationship between loneliness and self-reported health, controlling for demographics, isolation, and depression. Brooks and colleagues ([24]; this issue) explored self-efficacy for sleep, sleep quality, and relapse rates for participants with alcohol dependency. The investigators analyzed multiple data points and actigraphy data.

Two studies focused on clinical applications of sleep promotion interventions. In a randomized treatment outcome study, Fox and colleagues ([25]; this issue) examined relative rates of nighttime awakenings (per actigraph), selfreport sleep quality, and depression between bright light 
therapy and a dim light treatment. In another study, Irish and colleagues ([26]; this issue) evaluated whether participants' adherence to a self-help sleep promotion intervention related to an increase in sleep duration and other improvements in sleep health.

Three studies focused on underserved populations. April-Saunders and colleagues ([27]; this issue) studied cohorts of adolescents from Puerto Rico and from the South Bronx, New York. These researchers examined childhood adversities and sleep disturbance across three time points among 10 to 16-year-olds. Gatson and colleagues ([28]; this issue) investigated childhood experiences and sleep experience among Hispanic, Non-Hispanic White, and Black women. A focus of this article is the relationship of childhood trauma with sleep health across ethnic backgrounds. Characteristics of strength and resilience among Black women framed the third study in this group by McLaurin-Jones and colleagues ([29]; this issue). This study adds to the growing literature on stress and sleep disturbance in that there are unique stressors faced by Black women.

Two studies looked at sleep and immune response. Tucker and colleagues ([30]; this issue) considered the role of self-reported and actigraphyically assessed sleep in cancer survivors and found and increase in sleep time corresponded with a reduction in emotional distress and attenuation of pro-inflammatory biomarkers. In a timely paper, given the current pandemic, Prather and colleagues ([31]; this issue) investigated the role of sleep in promoting antibody production in response to flu immunizations. The implications of this study are provocative in the context of a world-wide process of immunization.

\section{Future Developments}

Although these articles represent a breadth of topics, problems, and methodologies, there are gaps in our selection worth noting. Although our initial response to a call for papers included studies from eight countries, only two studies in this issue were from outside the USA (i.e., Australia and Canada). In a related limitation, only three studies focused on underserved populations, resulting in a lack of representation of non-Black minorities, and no study focused on other marginalized groups such as sexual minorities. Unfortunately, none of the articles in this issue included a focus on sleep related breathing disorders or on circadian rhythms. The advantage of planning a second special issue on sleep is the opportunity to fill such gaps.

It is gratifying to showcase these articles that demonstrate the contribution of behavioral medicine in understanding and promoting sleep and wellbeing. Special issues provide an opportunity to reflect on accomplishments in the field, and they are also an appropriate venue for considering new directions and approaches.

Technological developments make it possible, and increasingly cost-effective, to collect enormous amounts of information, be that through online surveys, actigraphs, or physiological assessments, such as heart-rate. The development of home-based EEG assessment that is reliable, cost effective, and patient friendly cannot be far away. Advancing technologies in sleep assessment that are portable, less intrusive, and easy to use, such as increasingly reliable and valid smart watches, will promote ecological validity, encourage researchers to consider the influence of circadian rhythms, and generate a more comprehensive and detailed understanding of sleep and sleep-health relationships. Our field will advance as we emphasize sleep assessments that are multimodal, integrative, and prospective and when we are less satisfied with conducting single administration, cross-sectional surveys.

With an increasing ability to generate large data sets, it will perhaps be even more tempting to look inductively for associations that describe but not explain relationships (so-called dustbowl empiricism) [32]. As many of the articles in this issue illustrate, the stronger contributions to the field of sleep research are studies that progress beyond description to theory-based investigation. We anticipate that future contributions to the field will increasingly use experimental and quasi-experimental designs to evaluate sleep mechanisms and the efficacy of interventions.

Clearly, one new area of investigation is the need to study pandemic-related sleep changes, as people across the world have been forced to accommodate decreased mobility, increased social isolation, and changes to routines, roles, and social structures [33]. Also of consideration is the need to retool in order to conduct behavioral sleep science and to provide clinical services in a pandemic environment [34]. (Articles included in this review predate the COVID-19 pandemic.) How the pandemic has changed sleep and the long-term consequence of such changes will likely be a topic for behavioral medicine research for many years. Prather and colleagues' article in this issue may be prescient with immediate relevance to individual's response to vaccines for the COVID-19 virus.

As the pandemic experience has made clear, underserved groups are the least likely to receive adequate health care [35]. We are challenged with the need to tailor sleep promotion interventions to minority populations and to promote accessibility to health providers, most notably through internet access and the technologies to engage it. The development of evidence-based and telehealth sleep programs is likely to be increasingly in demand during and after the COVID-19 pandemic. Fortunately, such interventions have been in development for several years. For example, Williams and colleagues describe a telehealth 
intervention to improve sleep health among Black men and women at-risk for obstructive sleep apnea [36]. Further research is needed to understand how to deliver and sustain this intervention over time among underserved populations.

In closing, we hope that this special issue increases your appreciation of the novel and diverse ways sleep is understood by our field and that future research and interventions build on the lessons learned from studies included in this issue. We want to express our heartfelt appreciation to editors Michael Hoyt and Tracey Revenson for their guidance and the Society for Behavioral Medicine Sleep Special Interest Group for their support of this special issue. We look forward to a second such issue.

\section{Compliance with Ethical Standards}

Conflict of Interest The authors declare that they have no conflict of interest.

Ethical Approval For this type of paper, formal ethical review is not required.

Informed Consent This article does not report any new research data and therefore no consent was necessary.

Research Involving Human and Animal Participants This study does not present new data regarding studies with human participants.

Disclosure In the interest of full disclosure both authors are past chairs of the Society for Behavioral Medicine's Sleep SIG.

\section{References}

1. Trevorrow T. Sleepless in Honolulu: appreciating sleep problems across the developmental spectrum. Chaminade University Faculty In-service. 2018.

2. Roth T, Drake C, Roehrs. Behavioral sleep medicine. J Clin Sleep Med. 2013.

3. Kredlow MA, Capozzoli MC, Hearon BA, Calkins, AW, Otto, MW. The effects of physical activity on sleep: a meta-analytic review. J Behav Med. 2015

4. Smith MT, Haythornthwaite JA. How do sleep disturbance and chronic pain inter-relate? Sleep Med Rev: Insights from the longitudinal and cognitive-behavioral clinical trials literature; 2004.

5. Bayon V, Leger D, Gomez-Merino D, Vecchierini MF, Chennaoui M. Sleep debt and obesity. Annals Med. 2014;46(5):264-72.

6. Hasler BP, Smith LJ, Cousins JC, Bootzin RR. Circadian rhythms, sleep, and substance abuse. Sleep Med Rev. 2012;16(1):67-81.

7. Hysing M, Sivertsen B, Stormark K. Sleep in children with chronic illness, and the relation to emotional and behavioral problems-a population-based study. J Ped Psy. 2009;34(6):665-70.

8. American Psychiatric Association. Diagnostic and Statistical Manual of Mental Disorders. 5th ed. Arlington: VA. Am Psychiatric Assoc; 2013.

9. National Sleep Foundation. Sleep in America poll 2020. Langer Research Associates.
10. Peppard PE, Young T, Barnet JH, Palta M, Hagen EW, Hia KM. Increased prevalence of sleep-disordered breathing in adults. Am J Epidem. 2013;177(9):1006-14.

11. Trevorrow T, Zhou ES, Dietch JR, Gonzalez BD. Position statement: start middle and high schools at 8:30 am or later to promote student health and learning. Tran Beh Med. 2019;9(1):167-9.

12. Center for Disease Control and Prevention. Short sleep duration among middle and high school students-United States, 2015. MMWR. 2018;67(3).

13. Lin L, Wang J, Ou-yang X, Miao Q, Chen R, Liang F, Zhang Y, Tang Q. The immediate impact of the 2019 novel coronavirus (COVID-19) outbreak on subjective sleep status. Sleep Med. 2020 June.

14. Xiao H, Zhang Y, Kong D, Li S, Yang N. Social capital and sleep quality in individuals who self-isolate for 14 days during the Coronavirus disease 2019 (COVID-19) outbreak in January 2020 in China. Med Sci Monit. 2020;26:e923921-1.

15. Stanton E, Khalesi S, Williams SL, Alley SJ, Thwaite TL, Vandelanotte C. Depression, anxiety and stress during COVID19: associations with changes in physical activity, sleep, tobacco and alcohol use in Australian adults. Int J Environ Res Pub Health. 2020;17(11):1-13.

16. Casagrande M, Favieri F, Tambelli R, Forte G. The enemy who sealed the world: effects quarantine due to the COVID-19 on sleep quality, anxiety, and psychological distress in the Italian population. Sleep Med. 2020;75:12-20.

17. Huang Y, Zhao N. Generalized anxiety disorder, depressive symptoms and sleep quality during COVID-19 outbreak in China: a web-based cross-sectional survey. Psychiat Res. 2020;288.

18. Panjwani AA, Millar BM, Revenson TA. Tolerating uncertainty in the dark: insomnia symptoms, distress, and well-being among parents of adolescents and young adults with cancer. Int J Behav Med. In press.

19. Allen HK, Barrall Al, Vincent KB, Arria AM. Stress and burnout among graduate students: the buffering effects of sleep duration and quality. Int J Behav Med. In press.

20. Bunjo LJ, Reynolds AC, Appleton SL, Dorrian J, Vetter C, Gill TK, Adams RJ. Sleep duration moderates the relationship between perceived work-life interference and depressive symptoms in Australian men and women from the North West Adelaide health study. Int J Behav Med. In press.

21. Haynes. PL, Wolf, R1, Howe GW, Kelly MR. Unemployed individuals reporting hindrance work stress at previous job have increased likelihood of insomnia disorder. Int J Behav Med. In press.

22. Okun M, Obetz, V, Feliciano L. Subjective and objective sleep and cytokines in early pregnancy: association with pregnancy outcomes. In J Behav Med. In press.

23. Griffen SC, Mladen SN, Williams AB, Dautovich ND, Lageman SK, Dzierzewski JM, Perrin PB, Rybarczyk BD. Sleep disturbance mediates the association between loneliness and health in older Americans. In J Behav Med. In press.

24. Brooks AT, Kazmi N, Yang L, Tuason RT, Krumlauf MC, Wallen GR. Sleep-related cognitive/behavioral predictors of sleep quality and relapse in individuals with alcohol use disorder. In J Behav Med. In press.

25. Fox RS, Baik SH, McGinty H, Garcia SF, Reid KJ, Bovbjerg K, Fajardo P, Wu LM, Shahabi S, Ong JC, Zee PC, Penedo FJ. Feasibility and preliminary efficacy of a bright light intervention in ovarian and endometrial cancer survivors. In J Behav Med. In press.

26. Irish LA, Veronda AC, van Lamsweerde AE, Mead MP, Wonderlich SA. The process of developing a sleep health improvement plan: a lab-based model of self-help behavior.

27. April-Sanders A, Duarte CS, Wang S, McGlinchey E, Alcántara C, Bird H, Canino G, Suglia SF. Childhood adversity and sleep disturbances: longitudinal results in Puerto Rican children. In J Behav Med. In press. 
28. Gatson SA, McWhorter KL, Parks CG, D’Aloisio AA, RojoWissar DM, Sandler DP, Jackson CL. Racial/ethnic disparities in the relationship between traumatic childhood experiences and suboptimal sleep dimensions among adult women: findings from the Sister Study. In J Behav Med. In press.

29. McLaurin-Jones TL, Anderson AS, Marshall VJ, Lashley MB, Carter-Nolan PL. Superwomen and sleep: an assessment of Black college women across the African Diaspora. In J Behav Med. In press.

30. Tucker JA, Osann K, Hsieh S, Wahi A, Monk BJ, Wenzel L, Nelson EL. Longitudinal changes in sleep: associations with shifts in circulating cytokines and emotional distress in a cancer survivor population. In J Behav Med. In press.

31. Prather A, Pressman SD, Miller GE, Cohen SC. Temporal links between self-reported sleep and antibody responses to the influenza vaccine. Int J Behav Med. In press.

32. Latham, GP. 1925-1950 Dust bowl empiricism. In: Chapter 2. Work motivation: history, theory, research and practice. Sage Books.
33. Brooks A, Thornton L, Allen H, Trevorrow T. Behavioral medicine challenges in the shadow of a global pandemic. Trans Behav Med. In press.

34. Emanuel EJ, Persad G, Upshur R, Thome B, Parker M, Glickman A, Phillips JP. Fair allocation of scares medical resources in the time of Covid-19. New Eng J Med. 2020;382(21):2049-55.

35. Williams NJ, Robbins R, Rapoport D, Allegrante JP, Cohall A, Ogedgebe G, Jean-Louis G. Tailored approach to sleep health education (TASHE): study protocol for a web-based randomized controlled trial. Trials. 2016;17(1):585.

36. National Institute of Health-National Library of Medicine. Health Services Research Project in Progress. Nih/gov/ view_hsproj_record/20184095.

Publisher's Note Springer Nature remains neutral with regard to jurisdictional claims in published maps and institutional affiliations. 